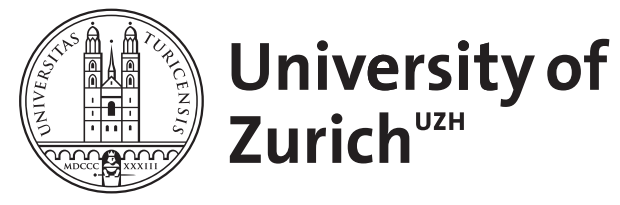

Zurich Open Repository and Archive

University of Zurich

University Library

Strickhofstrasse 39

CH-8057 Zurich

www.zora.uzh.ch

Year: 2019

\title{
Sozio-ökonomische Ungleichheit und konventionsrechtliche \\ Diskriminierungsverbote
}

Altwicker, Tilmann

Posted at the Zurich Open Repository and Archive, University of Zurich

ZORA URL: https://doi.org/10.5167/uzh-186098

Journal Article

Originally published at:

Altwicker, Tilmann (2019). Sozio-ökonomische Ungleichheit und konventionsrechtliche Diskriminierungsverbote. Juridikum, 6(2):237-247. 


\section{Sozio-ökonomische Ungleichheit und konventionsrechtliche Diskriminierungsverbote}

https://doi.org/10.33196/juridikum201902023701

\section{Tilmann Altwicker}

\section{Einleitung}

Die wirtschafts- und sozialwissenschaftliche Lit lässt kaum einen Zweifel daran zu, dass sozio-ökonomische Ungleichheit, oft vereinfachend als „Schere zwischen Arm und Reich“ bezeichnet, in best Staaten seit den 1970er-Jahren zunimmt. ${ }^{1}$ Für Deutschland wurde beispielsweise konstatiert, dass Aufstiege aus der Armut selten geworden seien. ${ }^{2}$ Innerstaatlich ist sozio-ökonomische Ungleichheit längst ein politisches Dauerthema. ${ }^{3}$ Auf der internationalen Ebene war dies bislang nicht der Fall: Während der Kampf gegen extreme Armut bereits seit den 1990er-Jahren einen Schwerpunkt internationaler Politik (insb der UN) einnimmt, beginnen Probleme sozio-ökonomischer Ungleichheit erst kürzlich verstärkt internationale Aufmerksamkeit zu erlangen. ${ }^{4}$ Wegweisend wirkt hierbei insb die Agenda 2030 für Nachhaltige Entwicklung der UN, die erstmals international das Ziel der Verringerung von Ungleichheit in und zwischen Staaten formuliert hat. ${ }^{5}$ Dieser Prozess wird inspiriert von der wachsenden Kritik an einer unzureichend gesteuerten wirtschaftlichen Globalisierung, ${ }^{6}$ der Kritik am Neoliberalismus ${ }^{7}$ sowie von Ansätzen zu einer alternativen, transnationalen Rechtspolitik. ${ }^{8}$ Von der Völkerrechtswissenschaft wird diese Entwicklung etwa im Rahmen einer sozialen Deutung des globalen Konstitutionalismus untersucht. ${ }^{9}$

1 Vgl Piketty, Das Kapital im 21. Jahrhundert (2013) 31; vgl auch die Nachweise bei Alston, Report of the Special Rapporteur on extreme poverty and human rights, A/HRC/29/31 2015, Abs 8.

2 Vgl Chassé, Diskriminierung von Armen und sozial Ausgegrenzten, in Scherr/El-Malfaalani/Yüksel (Hrsg), Handbuch Diskriminierung (2017) 479 (481).

3 Vgl etwa in der Schweiz die erfolgreiche eidgenössische Volksinitiative „gegen die Abzockerei“, die den als überhöht empfundenen Vergütungen des obersten Managements von börsenkotierten Aktiengesellschaften Einhalt bieten soll, AS 2013 1303, 15.5.2013.

4 Zur Entwicklung vgl Doz Costa, Poverty and Human Rights: From Rhetoric to Legal Obligations - A Critical Account of Conceptual Frameworks, SUR - International Journal on Human Rights 2008, 81.

5 UNGA Res A/RES/70/1,21.10.2015, Ziel 10. Vgl vorher schon UNGA Res A/RES/66/288, 11.09.2012, Annex Abs 4.

6 Vgl Milanovic, Global Inequality: A New Approach for the Age of Globalization (2018).

7 Vgl Ostry/Loungani/Furceri, Neoliberalism: Oversold? Finance \& Development 2016, 38; Brown, Sacrificial Citizenship: Neoliberalism, Human Capital and Austerity Politics, Constellations 2016, 3.

8 Vgl Fischer-Lescano/Möller, Der Kampf um globale soziale Rechte (2012) 39, 51.

9 Vgl Peters, Global Constitutionalism: The Social Dimension, in Peters/Suami/Vanoverbeke/Kumm (Hrsg), Global Constitutionalism from European and East Asian Perspectives (2018) 277. 
Dass sozio-ökonomische Ungleichheit faktisch den Genuss best Menschenrechte beeinträchtigen kann, ist bekannt. So zeigt der UN-Berichterstatter für extreme Armut und Menschenrechte, Philip Alston, ua anhand empirischer Studien auf, dass starke Korrelationen zwischen sozio-ökonomischer Ungleichheit und Gewaltkriminalität in wohlhabenden Staaten und solchen mit mittleren Einkommen beständen, aber auch Zusammenhänge von Ungleichheit mit politischer Instabilität und dem Genuss sozio-ökonomischer und kultureller Rechte (wie Zugang zu Gesundheitsversorgung, Bildung). ${ }^{10} \mathrm{Zu}$ ergänzen ist, dass sozio-ökonomische Ungleichheit Auswirkungen auf die Rechtsdurchsetzung hat, da mittellose Personen weniger oft den Rechtsweg beschreiten. ${ }^{11}$

Welchen Beitrag können menschenrechtliche Diskriminierungsverbote zur Lösung der Probleme, die mit steigender sozio-ökonomischer Ungleichheit verbunden sind, leisten? Dieser Frage soll hier am Bsp der Diskriminierungsverbote nach der Europäischen Menschenrechtskonvention (EMRK) nachgegangen werden. ${ }^{12}$ Diesbezüglich wird nachfolgend die These begründet, dass einerseits einer konventionsrechtlichen Korrektur der innerstaatlichen Verteilung sozio-ökonomischer Güter best Grenzen gezogen sind, dass aber andererseits das Potential der Diskriminierungsverbote nach der EMRK idZ auch noch nicht voll ausgeschöpft ist. Diese These wird in folgenden Schritten begründet: Zunächst werden Verbindungen zwischen einflussreichen, philosophischen Theorien der Verteilungsgerechtigkeit gezogen sowie begründet, unter welchen Bedingungen sozio-ökonomische Ungleichheit zwischen Individuen überhaupt zu einem Anti-Diskriminierungsproblem werden kann. Sodann wird beleuchtet, wie Probleme sozio-ökonomischer Ungleichheit unter den Diskriminierungsverboten der EMRK zu behandeln sind. Abschließend geht es um das Entwicklungspotential, das die konventionsrechtlichen Diskriminierungsverbote aufweisen hinsichtlich der Behandlung der mit sozio-ökonomischer Ungleichheit verbundenen Probleme.

\section{Soziale und ökonomische Ungleichheit - Von der Verteilungsgerechtigkeit zum Anti-Diskriminierungsrecht}

Begrifflich geht es bei Fragen sozio-ökonomischer Ungleichheit um die Verteilung best Güter in einer Gesellschaft, wobei in der rechtlichen Betrachtung zunächst offen bleiben kann, um welche es sich genau handelt. ${ }^{13}$ Entscheidend ist die Ungleichheit in der Verteilung zweier Klassen von Gütern: „Soziale Ungleichheit“ bezieht sich auf die Verteilung

$10 \mathrm{Vgl}$ Alston, A/HRC/29/31 2015, Abs 26ff.

$11 \mathrm{Vgl} \mathrm{Gilles,} \mathrm{Class} \mathrm{Warfare:} \mathrm{The} \mathrm{Disappearance} \mathrm{of} \mathrm{Low-Income} \mathrm{Litigants} \mathrm{from} \mathrm{the} \mathrm{Civil} \mathrm{Docket,} \mathrm{Emory} \mathrm{Law} \mathrm{Journal}$ 2016, 1531.

12 Europäische Menschenrechtskonvention, 4.11.1950, ETS Nr 5, 213 UNTS 222.

13 Der Grund ist, dass es sich bei den Gleichheitsrechten um inhaltsoffene, modale Rechte handelt, die eine Art und Weise des hoheitlichen Handelns betreffen, vgl dazu Altwicker, Menschenrechtlicher Gleichheitsschutz (2011) 44f. Anders in der ethischen Diskussion, vgl Koller, Recht und soziale Verteilungsgerechtigkeit in soziologischer und ethischer Perspektive, in Baumgartner/Heinrich/Rebhahn/Sutter (Hrsg), Verteilungsgerechtigkeit im Recht (2017) 25 (45). 
politischer Macht (zB Zugang zu politischen Ämtern) sowie auf die Verteilung gesellschaftlich produzierter Güter (zB Zugang zu Gesundheitsversorgung, Bildung, Obdach). ${ }^{14}$ „Ökonomische Ungleichheit“ betrifft die Verteilung wirtschaftlicher (dh marktfähiger) Ressourcen (zB Einkommen, Eigentum, weitere Vermögenswerte). ${ }^{15}$ Anders als bei Armut, bei der es um das Problem fehlender materieller Grundsicherung des Einzelnen (unabhängig von anderen) geht, bezieht sich sozio-ökonomische Ungleichheit auf die relative Position des Einzelnen (oder Gruppen) zu anderen in der Gesellschaft.

Fragen der Entstehung und des richtigen staatlichen Umgangs mit sozio-ökonomischer Ungleichheit sind klassische Probleme philosophischer Theorien der Verteilungsgerechtigkeit. ${ }^{16}$ Für die menschenrechtliche Diskussion haben dabei John Rawls' zweiter Gerechtigkeitsgrundsatz sowie Amartya Sens und Martha Nussbaums Fähigkeitenansatz ( „capability approach“) wesentliche Impulse geliefert. ${ }^{17}$ Rawls` zweiter Gerechtigkeitsgrundsatz benennt die Bedingungen, unter denen soziale und ökonomische Ungleichheiten in einer Gesellschaft gerechtfertigt sind, und erblickt diese im Prinzip der fairen Chancengleichheit (betreffend Ämter und Positionen) und, diesem nachgeordnet, dem Differenzprinzip (betreffend Einkommen und Vermögen). ${ }^{18}$ Von Bedeutung ist, dass Rawls eine faire und nicht bloß eine formale Chancengleichheit verlangt; dies erfordert eine folgenbezogene Betrachtung, die auch subtilere Formen der Diskriminierung berücksichtigt. ${ }^{19}$ Weiterhin stellt das Differenzprinzip eine grundsätzliche Gleichverteilungsvermutung auf. ${ }^{20}$ Daraus folgt, dass jede Ungleichverteilung ökonomischer Güter einer Rechtfertigung bedarf, wobei der einzig mögliche Rechtfertigungsgrund in der Aufwertung der sozialen Mindestposition erblickt wird. ${ }^{21}$ Das Differenzprinzip legt ferner einen asymmetrischen Ansatz nahe, der bei ökonomischen Verteilungsfragen die bes Berücksichtigung der Auswirkungen auf die sozial Schlechtestgestellten verlangt. ${ }^{22}$ Ähnlich bedeutsam ist der Fähigkeitenansatz von Sen und Nussbaum. Entscheidender Unterschied zu Rawls ist der Fokus auf Fähigkeiten anstelle von Gütern. Mit dem Fähigkeitenansatz ist insb die Idee verbunden, Lebensqualität an den menschlichen Fähigkeiten zu messen, also daran, „was die Menschen tatsächlich zu tun und zu sein in der Lage sind. “23 Für das Anti-Diskriminierungsrecht ist bedeutsam, dass der Fähigkeitenansatz zunächst dazu dient, relevante Ungleichheiten zu identifizieren. ${ }^{24}$ Zudem erlaubt der Fähigkeitenansatz

$14 \mathrm{Vgl}$ Alston, A/HRC/29/31 2015, Abs 6.

$15 \mathrm{Vgl}$ Alston, A/HRC/29/31 2015, Abs 5.

16 Für einen Überblick vgl Kersting, Philosophische Theorien der Verteilungsgerechtigkeit: Eine Skizze, in Baumgartner/ Heinrich/Rebhahn/Sutter (Hrsg), Verteilungsgerechtigkeit im Recht (2017) 1.

17 Rawls, Gerechtigkeit als Fairneß: Ein Neuentwurf (2006) 78ff; Sen, The Idea of Justice (2010) 231ff; Nussbaum, Die Grenzen der Gerechtigkeit (2010) 103ff.

$18 \mathrm{Vgl} \mathrm{Rawls}$, Gerechtigkeit 78.

19 Vgl Altwicker, Gleichheitsschutz 410.

$20 \mathrm{Vgl} \mathrm{Rawls,} \mathrm{Eine} \mathrm{Theorie} \mathrm{der} \mathrm{Gerechtigkeit} \mathrm{(1998)} 96$.

$21 \mathrm{Vgl}$ Altwicker, Gleichheitsschutz 410; vgl auch Koller in Baumgartner/Heinrich/Rebhahn/Sutter 45.

$22 \mathrm{Vgl}$ Altwicker, Gleichheitsschutz $410 \mathrm{f}$.

23 Nussbaum, Grenzen 104.

24 Vgl Sen, Inequality Reexamined (1992) $12 \mathrm{ff.}$ 
eine empirische Messbarkeit, mit der sich anhand von Indikatoren feststellen lässt, wie erfolgreich ein Staat etwa bei der Beseitigung von Formen der Diskriminierung ist. ${ }^{25}$ Allerdings führt kein gerader Weg von philosophischen Theorien der Verteilungsgerechtigkeit zum positiven Recht. Zu bedenken ist zunächst ein grundsätzlicher Unterschied zwischen Rechtsproblemen und dem Gerechtigkeitsproblem: So beziehen sich Rechtsprobleme (schon wegen der Zuschreibung von Verantwortlichkeiten) primär auf die (Un-)Gleichheit von Behandlungen, während das primäre Bezugsobjekt der philosophischen Gerechtigkeitsdiskussion in Zuständen der Gleichheit/Ungleichheit besteht. ${ }^{26}$ Angesichts der politischen Realitäten in westlichen Verfassungsstaaten ist es nicht verwunderlich, dass die weitreichenden Konsequenzen aus Rawls ${ }^{`}$ Differenzprinzip für die staatliche Gesetzgebung in der Praxis noch kaum gezogen wurden. ${ }^{27}$ Ein wenig besser sieht es für Sens und Nussbaums Fähigkeitenansatz aus: So hat dieser Ansatz immerhin zu einem gewandelten Fokus innerhalb des UN Development Program geführt. ${ }^{28}$

\section{Sozio-ökonomische Ungleichheit und konventionsrechtliche Diskriminierungsverbote}

Die philosophische und politische Bedeutsamkeit der Probleme sozio-ökonomischer Verteilungsgerechtigkeit steht in einem gewissen Kontrast zu deren rechtlicher Erfassbarkeit, wie nachfolgend am Bsp der konventionsrechtlichen Diskriminierungsverbote gezeigt werden soll. Rechtlich betrachtet ist nicht die Ungleichheit zwischen Personen das Problem, sondern die qualifiziert benachteiligende Behandlung im Vergleich zu Personen in ähnlicher Lage, die nicht gerechtfertigt werden kann. ${ }^{29}$ Die Erfassung sozio-ökonomischer Ungleichheit unter den konventionsrechtlichen Diskriminierungsverboten (Art 14 und Art 1 ZP 12 EMRK) wirft insb drei komplexe dogmatische Fragen auf: das Problem der strukturellen Priorität der Freiheitsrechte im Kontext von Art 14 EMRK, das Problem der schwachen, „klassenbezogenen“ Differenzierungsgründe und das Problem eines folgenbezogenen Anti-Diskriminierungsrechts.

\subsection{Akzessorietät: Strukturelle Priorität der Freiheitsrechte im Kontext von Art 14 EMRK}

Der konventionsrechtlichen Handhabbarkeit der Probleme sozio-ökonomischer Ungleichheit steht eine gewisse strukturelle Priorität der Freiheitsrechte insb vor dem Dis-

25 Vgl Fukuda-Parr, The Metrics of Human Rights: Complementarities of the Human Development and Capabilities Approach, Journal of Human Development and Capabilities 2011, 73.

26 Vgl näher Altwicker, Gleichheitsschutz $402 \mathrm{ff}$.

27 Andere Ansätze sind deutlich offener für eine Rechtfertigung ökonomischer Ungleichheit: Vgl zB Gosepath, Zu Begründungen sozialer Menschenrechte, in Gosepath/Lohmann (Hrsg), Philosophie der Menschenrechte (1998) 146 (177).

$28 \mathrm{Vgl}$ Robeyns, The Capability Approach in Practice, The Journal of Political Philosophy 2006, 351.

29 Vgl Altwicker, Gleichheitsschutz 113. 
kriminierungsverbot aus Art 14 EMRK entgegen. Dies schlägt sich in der unselbständigen Natur des Diskriminierungsverbots aus Art 14 EMRK nieder, dessen sog Akzessorietät: Für die Anwendbarkeit des Diskriminierungsverbots ist nämlich erforderlich, dass der Regelungsbereich (,,ambit“) eines der übrigen Konventionsrechte berührt ist. ${ }^{30} \mathrm{Ob}$ wohl der EGMR in Fragen der Regelungsbereichsberührung verhältnismäßig großzügig ist und etwa schon die bloß „thematische Einschlägigkeit“ eines Freiheitsrechts genügen lässt, handelt es sich im vorliegenden Kontext doch um eine bedeutsame Hürde. ${ }^{31}$ Der Grund ist folgender: Die EMRK gewährleistet explizit nur bürgerliche und politische Rechte, nicht aber wirtschaftliche und soziale Rechte. ${ }^{32}$ Die zwei Ausnahmen - das Eigentumsrecht und das Recht auf Bildung - sind nicht im Kerntext, sondern im optionalen ersten ZP geregelt. ${ }^{33}$ Nicht immer werden Probleme sozio-ökonomischer Ungleichheit zugleich den Regelungsbereich von Freiheitsrechten betreffen. Erforderlich ist, dass ein Vertragsstaat negative Rechtsfolgen an die „Bedürftigkeit“ (Einkommenssituation, Obdachlosigkeit) oder die soziale Stellung einer Person knüpft ${ }^{34}$ und dass zugleich der Regelungsbereich eines konventionsrechtlichen Freiheitsrechts betroffen ist. Folgende, hypothetische Szenarien kommen hier beispielhaft in Frage: Art 14 EMRK dürfte einschlägig sein, wenn die Gewährleistung überragend wichtiger Konventionsrechte wie das Recht auf Leben (Art 2 EMRK) oder Schutz vor unmenschlicher oder erniedrigender Behandlung (Art 3 EMRK) aufgrund von Bedürftigkeit nicht möglich ist. ${ }^{35}$ Dies wäre etwa der Fall, wenn der Staat Bedürftigen jede Subventionierung lebenswichtiger Medikamente oder in Not geratenen Personen eine lebenswichtige Operation versagt. ${ }^{36}$ Der Anwendungsbereich des Diskriminierungsverbots wäre wohl ebenfalls eröffnet, wenn Eingriffe in das Recht auf Familienleben aus Art 8 EMRK (etwa der Entzug des elterlichen Sorgerechts) allein mit der Bedürftigkeit der Eltern begründet würden. ${ }^{37}$ Allerdings handelt es sich um hypothetische Fälle, da der EGMR solche Konstellationen bislang allein unter einer freiheitsrechtlichen Perspektive entschieden hat. Zu Recht hat der Richter Pinto de Albuquerque in einer abweichenden Meinung im Fall Garib v Niederlande dieses Vorgehen des EGMR kritisiert. ${ }^{38}$ Der Fall betraf Wohnbeschränkungen in einem

30 Der genaue Umfang der erforderlichen Betroffenheit ist umstritten, vgl Altwicker, Gleichheitsschutz $146 \mathrm{ff}$.

$31 \mathrm{Vgl}$ Peters/König, Das Diskriminierungsverbot, in Dörr/Grote/Marauhn (Hrsg), Konkordanzkommentar EMRK/GG ${ }^{2}$ (2013) $1301 \mathrm{Rn} 35$.

$32 \mathrm{Zu}$ den Hintergründen vgl Schmahl/Winkler, Schutz vor Armut in der EMRK? Archiv des Völkerrechts 2010, 405 (407).

33 Leitjen, Core Socio-Economic Rights and the European Convention on Human Rights (2018) 29 und passim.

34 Die Frage, inwiefern die Bedürftigkeit bzw die soziale Stellung überhaupt anerkannte Diskriminierungsgründe darstellen, wird unten behandelt.

35 Vorausgesetzt ist, dass einer oder mehrere anerkannte Diskriminierungsgründe (zB der der „sozialen Herkunft“ oder des „Vermögens“) einschlägig sind, dazu s unten 3.2.

36 EGMR 9.4.2013,13423/09, Mehmet Şenturk und Bekir Şenturk/Türkei. Für weitere Beispiele vgl Lavrysen, Poverty and Human Rights: A European Perspective, in Brems/van der Beken/Yimer (Hrsg), Human rights and Development (2015) 301 (307ff).

37 Vgl die abw Meinung des Richters Silvis im Fall EGMR 6.10.2015, 58455/13, N.P./Republik Moldau.

$38 \mathrm{Vgl}$ die abw Meinung des Richters Pinto de Albuquerque im Fall EGMR 6.11.2017, 43494/09, Garib/Niederlande. 
Stadtbezirk Rotterdams: Die Beschwerdeführerin erhielt für den Wohnbezirk ihrer Wahl keine Wohngenehmigung, ua weil sie keiner bezahlten Arbeit nachging. Die Anwendung des Diskriminierungsverbots aus Art 14 EMRK wäre hier grundsätzlich möglich gewesen, da der Regelungsbereich des Rechts auf Freizügigkeit (Art 2 ZP 4 EMRK) betroffen war. ${ }^{39}$ Dennoch entschied eine Richtermehrheit den Fall allein freiheitsrechtlich (mit dem Ergebnis, dass keine Verletzung festgestellt wurde). Die strukturelle Priorität der Freiheitsrechte zeigt sich eben auch gerade in dem problematischen Vorgehen des EGMR, auf eine separate Prüfung des Diskriminierungsverbots mit dem Hinweis zu verzichten, dass die vorzunehmenden Wertungen bereits im Rahmen der Freiheitsrechtsprüfung berücksichtigt würden. ${ }^{40}$

\section{2. „Soziale Herkunft“ und „Vermögen“: „Klassenbezogene“ Diskriminierungsgründe?}

Das Potential des konventionsrechtlichen Anti-Diskriminierungsrechts wird auch durch das oben erwähnte Erfordernis einer qualifizierten Benachteiligung begrenzt. Die Benachteiligung (im Vergleich zu Personen in ähnlicher Lage) ist dann „qualifiziert“, wenn sie an einen personenbezogenen Diskriminierungsgrund (wie zB den des Alters oder des Geschlechts) anknüpft. ${ }^{41}$ Insofern ist nochmals in Erinnerung zu rufen, dass das Anti-Diskriminierungsrecht nicht (ungerechte) Verteilungen sozio-ökonomischer Güter an sich zum Gegenstand hat, sondern - wenn überhaupt - das benachteiligende „Verteilen“ dieser Güter auf der Basis personenbezogener Merkmale.

Nun legt bereits der Wortlaut des Art 14 und des Art 1 ZP 12 EMRK eine Anwendung der Diskriminierungsverbote auf Probleme sozio-ökonomischer Ungleichheit nahe, indem die Diskriminierungsgründe der „sozialen Herkunft“ und des „Vermögens“ explizit als relevante, personenbezogene Merkmale aufgelistet werden. Eine an die soziale Stellung oder den Besitz einer Person anknüpfende, unterschiedliche Behandlung bei der Güterverteilung scheint, prima facie, ein wichtiges Einfallstor zu sein, um Probleme sozio-ökonomischer Ungleichheit rechtlich zu lösen. In der Tat handelt es sich der Sache nach um „klassenbezogene“ Unterscheidungsmerkmale, die grundsätzlich geeignet wären, bestimmte Probleme sozio-ökonomischer Ungleichheit in den Anwendungsbereich der Diskriminierungsverbote zu bringen. Allerdings sind bislang weder der Begriff der „sozialen Herkunft“ noch der des „Vermögens“ vom EGMR selbst näher bestimmt worden. Beide Diskriminierungsgründe nehmen bislang nur eine untergeordnete Rolle in der Entscheidungspraxis des EGMR ein.

Der Grund für die relative Bedeutungslosigkeit des Diskriminierungsgrunds der „sozialen Herkunft" könnte darin zu erblicken sein, dass der Begriff selbst zu vage für eine

39 EGMR 6.11.2017, 43494/09, Garib/Niederlande, para 104ff.

40 Altwicker, Gleichheitsschutz $143 \mathrm{mwN}$.

41 Dazu Altwicker, Gleichheitsschutz 178ff. 
Anwendung ist. ${ }^{42}$ Zudem scheint die Rsp einem Begriffsverständnis zuzuneigen, nach welchem unter „sozialer Herkunft“ der „ererbte soziale Status einer Person“43 oder die „Abstammung und Verwurzelung von Menschen [...], vor allem die soziale Stellung der Vorfahren " 44 zu verstehen ist. Es sind indes kaum Fälle denkbar, in denen der Staat nachteilige Rechtsfolgen an die soziale Herkunft in diesem eng verstandenen Sinne knüpfen würde. Andernorts wird allerdings eine etwas weitere Begriffsbestimmung vertreten: Soziale Herkunft beziehe sich auf die Position, die der/die Einzelne durch die Geburt in einer bestimmten sozialen Klasse oder Gemeinschaft (zB aufgrund von Ethnizität, Religion oder Ideologie) erworben habe oder auf die soziale Situation, wie Armut und Obdachlosigkeit. ${ }^{45}$ Soweit ersichtlich, hat der Diskriminierungsgrund der „sozialen Herkunft" aber bislang nur in wenigen Fällen des EGMR überhaupt eine Rolle gespielt: Dabei wird zunächst deutlich, dass der EGMR den Diskriminierungsgrund der „sozialen Herkunft“ eng und im Zusammenhang mit dem ebenfalls explizit genannten Diskriminierungsgrund der „Geburt“ interpretiert. So ging es im Fall Stjerna darum, dass eine Behörde dem Beschwerdeführer verweigert hatte, den Namen eines vor über 200 Jahren verstorbenen Vorfahren anzunehmen. ${ }^{46}$ Der Beschwerdeführer sah sich hier in seiner sozialen Herkunft betroffen, weil einer seiner Vorfahren aus einer unehelichen Beziehung stammte. Da dies für die Verweigerung der Namensänderung durch die Behörden aber keine Rolle gespielt hatte, ging der EGMR hierauf nicht näher ein. Eine gewisse sozio-ökonomische Komponente weist der Fall Dempsey auf. ${ }^{47}$ Hier rügte die Beschwerdeführerin die ungleiche Behandlung in Bezug auf unentgeltlichen Rechtsbeistand in einem Adoptionsrechtsstreit. Die Beschwerdeführerin und leibliche Mutter des Kindes sah das Diskriminierungsverbot dadurch als verletzt an, dass die im Vergleich wohlhabenderen, prospektiven Adoptiveltern zügiger als sie Zugang zu unentgeltlichem Rechtsbeistand hatten. ${ }^{48}$ Mangels Substantiierung trat der EGMR aber auf diese Rüge nicht ein und erklärte die Beschwerde für unzulässig. Auch sonst hatte die Rüge einer Diskriminierung auf der Basis der „sozialen Herkunft“ vor dem EGMR, soweit ersichtlich, keinen Erfolg. ${ }^{49}$

Der Begriff des „Vermögens“ lässt sich als die von den Vertragsstaaten anerkannten, subjektiven Rechte an Gütern verstehen, wobei man zwischen Sach- und Geldvermögen

$42 \mathrm{Vgl}$ Explanatory Report to the European Convention on Nationality 6.11.1997, ETS No 166, para 43.

43 So, für den UNO-Pakt I, UN Committee on Economic, Social and Cultural Rights, General Comment No 20 2.7.2009, E/C.12/GC/20, para 24.

44 So, für die EUGRCh, Hölscheidt in Meyer, Charta der Grundrechte der Europäischen Union (2014) Art 21 Rn 44.

$45 \mathrm{Vgl}$ European Union Agency for Fundamental Rights and Council of Europe, Handbook on European non-discrimination law (2018) 218.

46 EGMR 25.11.1994, 18131/91, Stjerna/Finnland.

47 EGMR 6.4.2000, 41382/98, Dempsey/Irland.

48 EGMR 6.4.2000, 41382/98, Dempsey/Irland, para 2.

$49 \mathrm{Vgl}$ auch EGMR 31.8.2010, 20121/04, Truta/Rumänien, para 45f. 
und immateriellen Vermögen unterscheiden kann. ${ }^{50}$ Nun muss man allerdings grundsätzlich fragen, ob dieser Diskriminierungsgrund, der sowohl in Art 14 als auch in Art 1 ZP 12 EMRK genannt wird, wirklich den weiteren Begriff des „Vermögens“ oder nicht vielmehr den engeren des „Eigentums“ meint. Während die französische Fassung ( „la fortune“) den weiten Begriff des Vermögens nahelegt, spricht die gleichermaßen verbindliche englische Fassung von "property”. Aus den Travaux Préparatoires geht hervor, dass die ursprüngliche englische Fassung, die von „fortune“ sprach, im März 1950 aufgrund eines englischen Vorschlags in "property “ geändert wurde. ${ }^{51}$ Soweit ersichtlich ist dieser Unterschied in den Sprachfassungen vom EGMR bislang nicht thematisiert worden. Differenzierungen aufgrund des Vermögens einer Person (etwa ein progressives Steuersystem) dürften in der überwiegenden Mehrzahl der Fälle gerechtfertigt sein. ${ }^{52}$ So hat der EGMR Beschwerden, die sich auf den Diskriminierungsgrund des Vermögens beriefen, bisweilen als nicht hinreichend substantiiert zurückgewiesen. ${ }^{53}$ In anderen Fällen hat der EGMR die Diskriminierung geprüft und verneint, oft ohne näher auf den Diskriminierungsgrund einzugehen. ${ }^{54}$ So wurde im Fall Gillow ua die unterschiedliche Behandlung von Immobilien anhand von Einheitsgrenzwerten gerügt. ${ }^{55}$ Die Immobilie des Beschwerdeführers war nicht - wie ein kleiner Prozentsatz teurer, eher von wohlhabenden Personen nachgefragten Häuser auf der Insel Guernsey - vom behördlichen System des „controlled housing “ ausgenommen. ${ }^{56}$ In diesem Fall gelangte der EGMR dazu, das System des „controlled housing“ für gerechtfertigt zu halten, da so sichergestellt würde, dass auch ärmere Bevölkerungsschichten Zugang zu einem Teil des Wohnungsmarktes hätten. Sodann hatte die frühere Europäische Kommission für Menschenrechte (EKMR) den Fall H.S. und D.M. zu beurteilen, in welchem es ua um die Frage ging, ob das Fehlen von unentgeltlicher Rechtspflege und einer Deckelung von Schadensersatzforderungen eine Verletzung des Diskriminierungsverbots auf der Basis des Vermögens sei. ${ }^{57}$ In diesem Fall anerkannte die EKMR, dass wohlhabende Personen zwar unter den meisten Umständen besser in der Lage seien, ihre Rechte zu verteidigen. ${ }^{58}$ Anderseits gestand die EKMR den Vertragsstaaten einen Spielraum bei der Ausgestaltung der unentgeltlichen Rechtspflege zu, der es auch gestatte, einzelne Prozessgegenstände (wie vorliegend eine Klage betreffend üble Nachrede) von deren Anwendungsbereich auszuneh-

\footnotetext{
50 So in Bezug auf die EUGRCh Hölscheidt in Meyer, GRChEU Art 21 Rn 49. Weiter European Union Agency for Fundamental Rights and Council of Europe, Handbook 218.

51 Council of Europe, Preparatory work on Article 14 of the European Convention on Human Rights, CDH (67) 3, 95.1967, 15 u 17.

52 So in Bezug auf die EUGRCh Hölscheidt in Meyer, GRChEU Art 21 Rn 14.

53 EGMR 14.2.2006, 53434/99, Paulow/Finnland.

54 EGMR 3.6.2013, 26367/10, Fürst von Thurn und Taxis/Deutschland.

55 EGMR 24.11.1996, 9063/80, Gillow/Vereinigtes Königreich, para 63ff.

56 EGMR 24.11.1996, 9063/80, Gillow/Vereinigtes Königreich, para 63ff.

57 EKMR 5.5.1993, 21325/93, H.S. und D.M./Vereinigtes Königreich, para 3; vgl auch den Fall EGMR 9.10.1979, 6289/73, Airey/Irland, para 29f (kein Eintreten des EGMR auf den Beschwerdegrund der Diskriminierung aufgrund des Vermögens).

58 EKMR 5.5.1993, 21325/93, H.S. und D.M./Vereinigtes Königreich, para 3.
} 
men. Die Deckelung von Schadensersatzforderungen sei schon deswegen nicht erforderlich, weil die Einkommenssituation der BeschwerdeführerInnen und Beklagten durch das streitentscheidende Gericht berücksichtigt würde. Schließlich rügten die Beschwerdeführer im Fall Mamatas ua die nicht hinreichende Differenzierung zwischen KleinanlegerInnen und GroßanlegerInnen und beriefen sich damit implizit auf den Diskriminierungsgrund des Vermögens (allerdings letztlich ohne Erfolg)..$^{59}$ In Einzelfällen hat der EGMR eine Verletzung des Diskriminierungsverbots festgestellt: Im Fall Chassagnou ua erblickte der EGMR eine Verletzung des Diskriminierungsverbots aufgrund des Vermögens darin, dass EigentümerInnen kleinerer Ländereien zur Mitgliedschaft in einer Jagdgenossenschaft verpflichtet waren, während diese Pflicht EigentümerInnen großer Parzellen nicht traf. ${ }^{60}$

Insgesamt zeigt sich, dass die - jedenfalls potentiell - „klassenbezogenen“ Diskriminierungsgründe der „sozialen Herkunft“ und des „Vermögens“ bislang keine große Rolle in der Rsp des EGMR spielen. Eine Definition der Diskriminierungsgründe sucht man in der Rsp des EGMR bislang vergeblich, der Gerichtshof scheint sie aber beide eng auszulegen. Insb wird die Gruppe der „Armen“ oder der „sozial benachteiligten Personen“ von diesen Diskriminierungsgründen bislang nicht als solche erfasst.

\subsection{Strukturelle Diskriminierung: Ungleiche Auswirkungen und fortwirkende Benach- teiligung aufgrund früherer Ungleichbehandlung}

Während philosophische Theorien der Verteilungsgerechtigkeit die (Un-)Gerechtigkeit der Zustände der Verteilung von Gütern zum Gegenstand haben, knüpft das menschenrechtliche Anti-Diskriminierungsrecht an ein dem Vertragsstaat zurechenbares Verhalten an. ${ }^{61} \mathrm{Um}$ in den Anwendungsbereich des menschenrechtlichen Anti-Diskriminierungsrechts zu gelangen, müssen sich ungerechte Zustände in der Verteilung sozio-ökonomischer Güter also als Folge qualifiziert benachteiligender Behandlung darstellen. Dies bereitet keine Probleme in Fällen sog direkter Diskriminierung, wenn also ausdrücklich bei der Verteilung sozio-ökonomischer Güter an ein personenbezogenes Differenzierungskriterium angeknüpft wird. ${ }^{62}$ Allerdings sind die Fälle selten, in denen eine Person zB explizit wegen ihres Geschlechts weniger Sozialleistungen erhält: Im Fall Konstantin Markin erblickte der EGMR eine Verletzung des Diskriminierungsverbots in der unterschiedlichen Behandlung von männlichem und weiblichem Militärpersonal in Bezug auf den Elternurlaub. ${ }^{63}$

59 EGMR 21.7.2016, 63066/14 ua, Mamatas ua/Griechenland (betreffend eine Umschuldung der Staatsschulden mit Nennwertrückgang der Anleihen ohne Zustimmung privater Investoren).

60 EGMR 29.4.1999, 25088/94, Chassagnou ua/Frankreich, para $118 \mathrm{ff}$.

$61 \mathrm{Vgl}$ Altwicker, Gleichheitsschutz $402 \mathrm{f}$.

62 Vgl Peters/Altwicker, Europäische Menschenrechtskonvention (2012) $246 \mathrm{f}$.

63 EGMR 22.3.2012, 30078/06, Konstatin Markin/Russland, para 152. 
Probleme sozio-ökonomischer Ungleichheit sind in der Praxis hingegen oft mit struktureller Diskriminierung verbunden, unter der hier die ungleiche Auswirkung an sich neutraler Behandlungen Einzelner sowie die fortwirkende Benachteiligung aufgrund vergangener Ungleichbehandlung verstanden werden sollen. Nur ein komplexes Anti-Diskriminierungsrecht kann diese beiden Phänomene erfassen. Unter der EMRK ist dies zumindest teilweise möglich. So ist seit einiger Zeit die Figur der indirekten Diskriminierung anerkannt, bei der die Ungleichbehandlung auf einem dem Anschein nach neutralen Differenzierungsgrund beruht, die aber eine geschützte Personengruppe unverhältnismäßig belastet und die nicht gerechtfertigt ist. ${ }^{64} \mathrm{Zu}$ beachten ist aber auch hier: Die unverhältnismäßige Belastung der Gruppe der „Armen“ durch eine an sich neutrale gesetzliche Regelung oder Verwaltungspraxis wäre gegenwärtig kein Problem des menschenrechtlichen Anti-Diskriminierungsrechts, da - wie dargelegt - diese derzeit keine geschützte Personengruppe bildet. Erfasst sind derzeit nur Fälle, in denen die unverhältnismäßig belastende Wirkung einer staatlichen Maßnahme bei bedürftigen Personen einer aus einem anderen Grund geschützten Personengruppe (zB der Gruppe der Roma, der alleinerziehenden Frauen) eintritt.

Unter der EMRK derzeit noch kaum entwickelt ist die fördernde Sonderbehandlung (engl „affirmative action“) von Angehörigen bestimmter sozialer Gruppen, die auf den Ausgleich faktischer, fortwirkender Benachteiligung abzielt. ${ }^{65}$ Grundsätzlich hat der EGMR aber anerkannt, dass der Staat uU verpflichtet sein kann, derartige Fördermaßnahmen vorzusehen. ${ }^{66}$

\section{Entwicklungspotential}

Das Entwicklungspotential der konventionsrechtlichen Diskriminierungsverbote in Bezug auf Probleme sozio-ökonomischer Ungleichheit ist noch nicht ausgeschöpft. Erstens kommt Art 1 ZP 12 EMRK für das Problem sozio-ökonomischer Ungleichheit eine potentiell große Relevanz zu. Durch Art 1 ZP 12 EMRK wurde ein allgemeines (dh nicht akzessorisches) Diskriminierungsverbot eingeführt, wodurch die oben diskutierte strukturelle Priorität der Freiheitsrechte abgemildert wird. Gerade Fälle der Ungleichbehandlung in sozio-ökonomischen Rechten, in denen sich bisher kein Zusammenhang mit den Freiheitsrechten herstellen ließ (womit infolgedessen Art 14 EMRK unanwendbar war), geraten mit Art 1 ZP 12 EMRK in den Fokus. ${ }^{67}$ Allerdings ist die Durchschlagskraft des allgemeinen Diskriminierungsverbots dadurch beschränkt, dass es bis heute (Stand: 19.2.2019) nur von 20 Vertragsstaaten ratifiziert wurde und in den übrigen Staaten nach wie vor große Bedenken gegen eine Ausweitung des konventionsrechtlichen Anti-Dis-

\footnotetext{
$64 \mathrm{Vgl}$ Altwicker, Gleichheitsschutz $266 \mathrm{ff}$.

$65 \mathrm{Vgl}$ Peters/Altwicker, Menschenrechtskonvention 250.

66 EGMR 24.4.2012, 25446/06, Yordanova ua/Russland, para 129.

$67 \mathrm{Vgl}$ Altwicker, Gleichheitsschutz 157-160.
} 
kriminierungsrechts bestehen. So wurde das 12. Zusatzprotokoll etwa von Deutschland und Ö zwar unterzeichnet, aber bislang nicht ratifiziert, im Fall der Schweiz fehlt es bereits an der Unterzeichnung.

Zweitens sollte die Rsp den Kreis der personenbezogenen Differenzierungsgründe in Art 14 und Art 1 ZP 12 EMRK um die Gründe „Armut“ bzw „sozio-ökonomischen Status“ erweitern. Wie beschrieben, bildet die soziale Gruppe der „Armen“ oder „Bedürftigen“ derzeit keine eigenständige, vom Diskriminierungsverbot geschützte Personengruppe. Eine Erweiterung ist möglich, da die Auflistung der Diskriminierungsgründe in Art 14 EMRK nicht abschließend ist. ${ }^{68}$ So wäre durchaus denkbar, entweder den Diskriminierungsgrund der „sozialen Herkunft“ weiter auszulegen oder, besser noch, den sozio-ökonomischen Status, die Armut oder die Bedürftigkeit einer Person als „sonstigen Status“ iSv Art 14 und Art 1 ZP 12 EMRK anzuerkennen. Nur so wäre sichergestellt, dass wesentliche Probleme sozio-ökonomischer Ungleichheit in den Anwendungsbereich der Diskriminierungsverbote gelangen können. Eine solche Erweiterung stände im Einklang mit Vorstößen, Fragen sozio-ökonomischer Ungleichheit justiziabler zu machen. So hat der Europäische Ausschuss für soziale Rechte (ECSR) kürzlich in einer Beschwerde betreffend den sozialen Wohnungsbau in Irland angedeutet, dass ua auch der sozioökonomische Status bzw die Bedürftigkeit als Diskriminierungsgründe anzuerkennen seien ${ }^{69}$ In diesem Sinne hat Richter Pinto de Albuquerque auch zu Recht kritisiert, dass der EGMR im Fall Garib v Niederlande eine Gelegenheit hat verstreichen lassen, „Armut“ als weiteren Diskriminierungsgrund anzuerkennen. ${ }^{70}$ Den Vertragsstaaten würde aber wohl in jedem Fall ein weiter Beurteilungsspielraum in sozio-ökonomischen Verteilungsfragen eingeräumt werden müssen. ${ }^{71}$ Eine zeitgemäße Auslegung des Diskriminierungsverbots verlangt jedoch, eklatante Ungerechtigkeiten in der Verteilung sozioökonomischer Güter durch den Staat auch auf völkerrechtlicher Ebene justiziabel zu machen.

Tilmann Altwicker hat eine SNF-Förderungsprofessur für Öffentliches Recht, Völkerrecht, Rechtsphilosophie und Empirische Rechtsforschung an der Rechtswissenschaftlichen Fakultät der Universität Zürich inne; tilmann.altwicker @rwi.uzh.ch.

$68 \mathrm{Vgl}$ Peters/Altwicker, Menschenrechtskonvention 245.

69 ECSR 12.5.2017, 110/2014, International Federation for Human Rights (FIDH)/Irland, para 124.

$70 \mathrm{Vgl}$ die abw Meinung des Richters Pinto de Albuquerque im Fall EGMR 6.11.2017, 43494/09, Garib/Niederlande, para $25 \mathrm{ff}$.

71 Zuletzt EGMR 7.2.2019, 37115/11, Yeshtla/Niederlande, para 39 (betreffend den Entzug von Wohngeld). 